\title{
Induced Genetic Variability and Scope of Selection for Yield Attributes in Greengram (Vigna radiata L. Wilczek)
}

\author{
Tapas Ranjan Das ${ }^{1 *}$, Bhabendra Baisakh ${ }^{2}$ and Arjun Mohan Prusti ${ }^{2}$ \\ ${ }^{1}$ ICAR- IARI Regional station, Pusa, Samastipur, Bihar-848125, India \\ ${ }^{2}$ Deptt. of Plant Breeding and Genetics, OUAT, Bhubaneswar, Odisha, 751 003, India \\ *Corresponding author
}

\section{A B S T R A C T}

\section{Keywords}

Genetic variability, Greengram, Induced mutation, Mutagens, Yield

\section{Article Info}

Accepted:

22 March 2020

Available Online:

10 April 2020
An experiment was conducted to generate a broad genetic variability through induced mutation using physical and chemical mutagens. Three doses each of gamma rays $(20,40$ and $60 \mathrm{kR})$, ethyl methane sulphonate $(0.2,0.4$ and $0.6 \%)$, nitrosoguanidine $(0.005,0.010$, and $0.015 \%$ ), maleic hydrazide $(0.01,0.02$ and $0.03 \%$ ) and their combinations were administered to the seed of two greengram varieties, Sujata and OBGG-52. In $\mathrm{M}_{2}$ generation most treatment populations exhibited a reduction in population mean and increased in population variance for pods/plant, seeds/pod, 100 seed weight, yield/plant and the magnitude of such changes varied with mutagens, their doses and genotypes. A greater shift in mean and variance was observed in treatments with higher doses. The negative shift of mean was more pronounced in $\mathrm{MH}$ and its combined treatments in both varieties. In general, most of the mutagen treated populations showed a wider range of variation than the parent varieties and the variation was in both directions. The higher values of heritability and genetic advance with a high genotypic coefficient of variation for most of the yield attributing traits confirmed that selection in $\mathbf{M}_{2}$ populations would be effective in bringing the improvement in yield/plant and its direct components like pods/plant, seeds/pod and 100 seed weight.

\section{Introduction}

Greengram [Vigna radiata (L.) Wilczek] is one of the most important pulse crops, grown from tropical to sub-tropical areas around the world. It generates a triple benefit:income, additional nutrient-rich food, and increased soil fertility via biological nitrogen fixation. Greengram is grown on more than six million ha worldwide (about $8.5 \%$ of global pulse area). India is the world's largest producer as well as consumer of greengram. Though India is the highest producer in the world, productivity is still very low i.e. $500 \mathrm{~kg} / \mathrm{ha}$ (Anonymous. 2018). The bottlenecks in its improvement have been the lack of variability in different traits and improvement of one trait on its own will affect the performance of other traits because of genotypic correlations between traits (Das and Baisakh, 2019). Since genetic variability is essential for crop improvement programme, induction of 
mutation by different physical and chemical mutagens provide a powerful means of creating new and useful variability in greengram both in qualitative and quantitative traits (Das et al., 2006). Information on the extent of induced polygenic variability and the genetic parameters for different polygenic traits in $\mathbf{M}_{2}$ generation indicates the scope of improvement in traits through selection. Thus the present study was undertaken to generate a broad genetic variability through induced mutation using physical and chemical mutagens and study the effect of different mutagens, ascertain the magnitude of induced genetic variability and other genetic parameters of yield and its components in $\mathrm{M}_{2}$ generation.

\section{Materials and Methods}

Healthy seeds of two greengram varieties namely Sujata and OBGG-52 were administered mutagenic treatments with three doses each of gamma rays (20, 40 and $60 \mathrm{kR})$, ethyl methane sulphonate (EMS) $(0.2,0.4$ and $0.6 \%)$, nitroso guanidine $(\mathrm{NG})(0.005,0.010$ and $0.015 \%$ ) and maleic hydrazide $(\mathrm{MH})$ $(0.01,0.02$ and $0.03 \%)$ singly and combine mutagens of $40 \mathrm{kR}$ gamma rays with $0.4 \%$ EMS or $0.010 \% \mathrm{NG}$ or $0.02 \% \mathrm{MH}$. The details of mutagenic treatments and the symbols used for treatments are presented in Table 1.

Dry seeds were irradiated with gamma rays treatment at Bhaba Atomic Research Centre (BARC), Trombay (India). For treatment with EMS, NG, and MH, the seeds were presoaked in distilled water for six hours, blotted dry and then treated with a freshly prepared aqueous solution of above chemical mutagens for six hours, with intermittent shaking. For combination treatments, seeds were first irradiated with $40 \mathrm{kR}$ gamma rays and then treated with $0.4 \%$ EMS or $0.01 \% \mathrm{NG}$ or $0.02 \% \mathrm{MH}$ solution in the same manner as described above. After treatment, the seeds were thoroughly washed with running water to bleach out the residual chemicals and then dried on blotting paper after treatment. These treated seeds were sown as $\mathrm{M}_{1}$ and the seed of $M_{1}$ used to grow the $M_{2}$ generation at Orissa University of Agriculture and Technology, Bhubaneswar, in two separate trials in randomized block design (RBD) with three replications and spacing of $25 \times 10 \mathrm{~cm}^{2}$. Twenty normal-looking plants, excluding the macro-mutants, were randomly chosen from each plot and observations were recorded on yield/plant (g) and yield attributing traits. The mean and variance of the traits in each treatment population were estimated and subjected to statistical analysis. The genetic parameters for the traits in different mutagenic treatment populations were calculated as follows:

Genotypic variance $\left(\sigma_{\mathrm{g}}^{2}\right)=\sigma_{\mathrm{p}}^{2}-\sigma_{\mathrm{e}}^{2}$

Phenotypic coefficient of

variability $(\mathrm{PCV}) \quad=\frac{\sigma_{p}}{\text { Mean }} \times 100$

Genotypic coefficient of

variability $(\mathrm{GCV}) \quad=\frac{\sigma_{\mathrm{g}}}{\text { Mean }} \times 100$

Heritability coefficient $\left(h^{2}\right)=\frac{\sigma_{\mathrm{g}}^{2}}{\sigma_{\mathrm{p}}^{2}}$

Heritability $\left(\mathrm{h}^{2}\right.$ in \%) $\quad=\frac{\sigma_{\mathrm{g}}^{2}}{\sigma_{\mathrm{p}}^{2}} \times 100$

Genetic Advance $(\mathrm{GA}) \quad=\mathrm{k} \cdot \mathrm{h} \cdot \sigma_{\mathrm{g}}=\mathrm{k} \cdot \mathrm{h}^{2} \cdot \sigma_{\mathrm{p}}$

GA (as $\%$ of mean $) \quad=\frac{\text { GA }}{\text { Mean }} \times 100$

Where,

$\mathrm{k}=\quad$ Standardized selection differential for specified selection intensity at $5 \%$.

$\mathrm{h}=\quad$ Square root of heritability coefficient

$\sigma_{\mathrm{g}}=$ Square root of genotypic variance 
$\sigma_{p}=$ Square root of phenotypic variance

\section{Results and Discussion}

To assess the nature and magnitude of induced polygenic variability of micro mutations in quantitative traits like pods/plant, seeds/pod, 100-seed weight and yield/plant of the different mutagenic treatments, populations of $\mathrm{M}_{2}$ generation were analyzed through statistical parameters such as mean, variance, heritability and genetic advance. Analysis of variance for pods/plant, seeds/pod, 100-seed weight and yield/plant of $\mathrm{M}_{2}$ population showed significant differences among the treatments for the above characters in both the varieties. The effects of different doses of different mutagen on different genetic parameters and in the induction of variability in different quantitative characters are as follows:

\section{Pods per plant (Table 2)}

The population means for pods/plant in different treatments of Sujata and OBGG-52 varied from 7.4 to 9.50 and 6.45 to 8.48 , respectively, while in their respective controls it was 9.52 and 8.55. All mutagenic treatments exhibited lower pods/plant than respective controls. The negative shift in mean was significant for E3, M1, M2, M3 in Sujata and E3, M1, M2, M3, GM2 in OBGG52 . The reduction in mean generally increased with mutagen dose and the reduction was more pronounced in EMS and MH treatments in Sujata. The ranges of variation for pods per plant in the control populations were 7 to 13 in Sujata and 6 to 11 in OBGG-52. The variations in different treated populations for pods/plant were observed 3 to 16 in the case of Sujata and 4 to 15 in OBGG-52. Though the induced variation in pods/plant was in both directions, the lower mean of most treatment populations indicated the induction of more negative micro mutations. The variance of pods/plant in treated populations of Sujata and OBGG-52 ranged from 3.85 to 7.46 and 1.94 to 6.21 as against 1.82 and 1.77 in the respective controls. All the mutagenic treatments exhibited greater population variance than controls in Sujata whereas a significant increase of variance over control was observed in all mutagenic treatments except MH treatments and GN2 in OBGG-52. The PCV estimates in the different $\mathrm{M}_{2}$ populations varied from $20.65 \%$ (N1) to $33.11 \%$ (E3) in Sujata and $21.59 \%$ (M3) to $32.65 \%$ (E3) in OBGG-52. The GCV estimates in the different $\mathrm{M}_{2}$ populations varied from $15.00 \%$ (N1) to $28.64 \%$ (E3) in Sujata and $6.39 \%$ (M3) to $26.65 \%$ (E3) in OBGG-52.

Heritability estimates for the trait in the treated populations varied from $52.73 \%(\mathrm{~N} 1)$ to $75.60 \%(\mathrm{G} 3)$ and $8.76 \%(\mathrm{M} 3)$ to $71.50 \%$ (E1, E2) in Sujata and OBGG-52, respectively. Genetic advance (GA) at 5\% selection intensity in the treatments varied from $2.13(\mathrm{~N} 1)$ to $4.25(\mathrm{G} 3)$ in Sujata and 0.25 (M3) to 3.67(E1, E2) in OBGG-52. GA as a percentage of mean ranged from 22.43 to 51.04 in Sujata and 3.90 to 44.92 in OBGG52. GA as a percentage of mean was higher for all treatments except N1 in Sujata and M2 and M3 in OBGG-52.

\section{Seeds per pod (Table 3)}

The mean value for seeds per pod in the treated population of Sujata varied from 7.01 to 8.27 and in OBGG-52 varied from 6.89 to 8.49 while in respective controls it was 8.55 and 8.51, respectively. All mutagenic treatments of both the varieties showed a lower mean than respective controls. The negative shift of mean was significant for G2, N2, N3, M1, M2, M3, GE2, GN2, GM2 in Sujata and M3, GE2, GN2, GM2 in OBGG52. Though there was no definite trend, the reduction was generally higher in $\mathrm{MH}$ and 
combined treatments. The range for seeds per pod in the parent population was 6.4 to 9.8 in Sujata and 7.4 to 9.6 in OBGG-52 while it was much wider in treated populations with a range of 3.5 to 10.5 in Sujata and 4.3 to 11.6 in OBGG-52. Though the induced variation was in both directions, it was more in a negative direction. The variance of seeds/pod in $\mathrm{M}_{2}$ populations of Sujata and OBGG-52 ranged from 0.89 to 2.22 and 0.54 to 1.53 , respectively, while population variance in respective controls were 0.76 and 0.28 . All treatments in both varieties showed higher population variance than respective controls. In Sujata, a significant increase of variance over control was observed in all mutagenic treatments except $\mathrm{G} 1$ and GN2. In case of OBGG-52, significant increase of variance over control was observed in G2, G3, E1, E2, E3, N3, M2, M3 and GN2. The PCV estimates in the different $\mathrm{M}_{2}$ populations varied from $12.02 \%$ (GN2) to $20.03 \%$ (M3) in Sujata and from $8.92 \%$ (M1) to $17.55 \%$ (GN2) in OBGG-52. The GCV estimates in the different $\mathrm{M}_{2}$ populations varied from $4.59 \%$ (GN2) to $16.24 \%$ (M3) in Sujata and from $6.19 \%(\mathrm{M} 1)$ to $15.79 \%(\mathrm{GN} 2)$ in case of OBGG-52.

The heritability for seeds/pod in treated populations was varied from $14.61 \%$ (GN2) to $65.77 \%$ (M3) and $48.15 \%(\mathrm{M} 1)$ to $81.70 \%$ (G3) in Sujata and OBGG-52, respectively. Heritability estimates were high in all the treatments except G1, GN2, and GM2 in Sujata and M1 in OBGG-52. GA estimates in treatments varied from 0.28 to 2.02 in Sujata and 0.73 to 2.08 in OBGG-52. GA as a percentage of mean showed a variation of $3.62 \%(\mathrm{GN} 2)$ to $27.13 \%(\mathrm{M} 3)$ and $8.85 \%$ (M1) to $29.26 \%$ (GN2) in Sujata and OBGG52 , respectively. The GA estimates for the trait in the treated populations were generally moderate.

100-seed weight (Table 4)
The populations mean values for the 100-seed weight $(\mathrm{gm})$ in treated populations of Sujata and OBGG-52 varied from 1.97 to $2.63 \mathrm{gm}$ and 2.73 to $3.09 \mathrm{gm}$, respectively, whereas in respective controls it was $2.67 \mathrm{gm}$ and $3.22 \mathrm{gm}$. The negative shift of mean in mutagenic treatments was significant for M2, M3, and GE2 in Sujata and all except G1 in OBGG-52. The reduction in mean generally increased with mutagen dose in both cases. The range of 100-seed weight in control populations of Sujata and OBGG-52 showed a range of variation of 2.28 to $2.96 \mathrm{gm}$ and 2.85 to $3.57 \mathrm{gm}$, respectively. The ranges of variation of 100 -seed weight in the treated populations of these two varieties were 1.52 to $3.54 \mathrm{gm}$ in Sujata and 2.1 to $3.8 \mathrm{gm}$ in OBGG-52. The variance of 100-seed weight in the treated populations varied from 0.053 to 0.1 in Sujata and 0.072 to 0.121 in OBGG52 , whereas in respective controls the variances were 0.03 and 0.05 . Increases in variance over control were significant in all mutagenic treated populations of both the varieties except for GE2 in Sujata and G1 and GM2 in OBGG-52. The PCV estimates for the trait in different treated populations of Sujata and OBGG-52 ranged from 8.65 (E1) to $11.72(\mathrm{G} 3)$ and $8.68(\mathrm{G} 1)$ to $12.41(\mathrm{M} 3)$, respectively. The GCV estimates for the trait in different treated populations of Sujata and OBGG-52 ranged from 5.05 (GE2) to $9.41(\mathrm{G} 3)$ and 4.8 (G1) to 9.45 (GE2), respectively.

The treated populations of Sujata and OBGG52 had heritability estimates of $27.27 \%$ (GE2) to $64.44 \%(\mathrm{G} 3)$ and $30.56 \%(\mathrm{G} 1)$ to $58.68 \%$ (M1), respectively. There were a lot of differences in heritability among the treatments and estimates were higher in all treatments of Sujata (except E1, M3, and GE2) and OBGG-52 (except G1 and GM2). Genetic advance in the treatments ranged from 0.12 (GE2) to $0.40(\mathrm{G} 3)$ and $0.17(\mathrm{G} 1)$ to 0.42 (M1, GE2) in Sujata and OBGG-52, 
respectively. Genetic advance as a percentage of mean varied from $5.43 \%$ (GE2) to $15.56 \%$
(G3) in Sujata and $5.47 \%$ (G1) to $14.87 \%$ (GN2) in OBGG-52.

Table.1 Details of mutagenic treatments

\begin{tabular}{|c|c|c|c|c|c|}
\hline $\begin{array}{l}\text { Tr. } \\
\text { No. }\end{array}$ & $\begin{array}{c}\text { Treatment } \\
\text { symbol }\end{array}$ & Mutagen & $\begin{array}{c}\text { Dose/ } \\
\text { concentration }\end{array}$ & $\begin{array}{c}\text { Duration of } \\
\text { pre-soaking } \\
\text { in distilled } \\
\text { water }\end{array}$ & $\begin{array}{l}\text { Duration of } \\
\text { treatment with } \\
\text { mutagenic } \\
\text { solution }\end{array}$ \\
\hline 1 & G1 & Gamma-rays & $20 \mathrm{kR}$ & - & - \\
\hline 2 & G2 & Gamma-rays & $40 \mathrm{kR}$ & - & - \\
\hline 3 & G3 & Gamma-rays & $60 \mathrm{kR}$ & - & - \\
\hline 4 & E1 & EMS & $0.2 \%$ & 6 hours & 6 hours \\
\hline 5 & E2 & EMS & $0.4 \%$ & 6 hours & 6 hours \\
\hline 6 & E3 & EMS & $0.6 \%$ & 6 hours & 6 hours \\
\hline 7 & N1 & NG & $0.005 \%$ & 6 hours & 6 hours \\
\hline 8 & $\mathrm{~N} 2$ & NG & $0.010 \%$ & 6 hours & 6 hours \\
\hline 9 & N3 & NG & $0.015 \%$ & 6 hours & 6 hours \\
\hline 10 & M1 & MH & $0.01 \%$ & 6 hours & 6 hours \\
\hline 11 & M2 & $\mathrm{MH}$ & $0.02 \%$ & 6 hours & 6 hours \\
\hline 12 & M3 & MH & $0.03 \%$ & 6 hours & 6 hours \\
\hline 13 & GE2 & \multicolumn{2}{|c|}{ Gamma-rays $40 \mathrm{kR}+$ EMS $(0.4 \%)$} & 6 hours & 6 hours \\
\hline 14 & GN2 & \multicolumn{2}{|c|}{ Gamma-rays 40 kR + NG $(0.010 \%)$} & 6 hours & 6 hours \\
\hline 15 & GM2 & \multicolumn{2}{|c|}{ Gamma-rays $40 \mathrm{kR}+\mathrm{MH}(0.02 \%)$} & 6 hours & 6 hours \\
\hline 16 & $\mathrm{C}$ & \multicolumn{2}{|l|}{ Control (parent) } & 6 hours & - \\
\hline
\end{tabular}

Table. 2 Genetic parameters for pods per plant in different mutagenic treatment in $\mathrm{M}_{2}$ population 
of greengram

\begin{tabular}{|c|c|c|c|c|c|c|c|c|c|}
\hline $\begin{array}{l}\text { Tr. } \\
\text { No. }\end{array}$ & $\begin{array}{l}\text { Tr. } \\
\text { Code }\end{array}$ & Range & Mean & Variance & $\begin{array}{l}\text { PCV } \\
(\%)\end{array}$ & $\begin{array}{c}\text { GCV } \\
(\%)\end{array}$ & $\begin{array}{c}h^{2} \\
(\%)\end{array}$ & $\begin{array}{c}\text { GA } \\
(5 \%)\end{array}$ & $\begin{array}{c}\text { GA (\% of } \\
\text { Mean) }\end{array}$ \\
\hline \multicolumn{10}{|c|}{ Sujata } \\
\hline 1 & G1 & $5-15$ & 9.47 & $5.91 *$ & 25.67 & 21.36 & 69.20 & 3.47 & 36.60 \\
\hline 2 & $\mathrm{G} 2$ & $5-15$ & 9.35 & $7.23 *$ & 28.76 & 24.88 & 74.83 & 4.14 & 44.33 \\
\hline 3 & G3 & $4-15$ & 8.80 & $7.46 *$ & 31.04 & 26.99 & 75.60 & 4.25 & 48.34 \\
\hline 4 & E1 & $5-15$ & 8.72 & $5.06 *$ & 25.80 & 20.64 & 64.03 & 2.97 & 34.03 \\
\hline 5 & E2 & $3-16$ & 8.67 & $7.20 *$ & 30.95 & 26.75 & 74.72 & 4.13 & 47.64 \\
\hline 6 & E3 & $3-16$ & $8.12 *$ & $7.23 *$ & 33.11 & 28.64 & 74.83 & 4.14 & 51.04 \\
\hline 7 & N1 & $4-14$ & 9.50 & $3.85 *$ & 20.65 & 15.00 & 52.73 & 2.13 & 22.43 \\
\hline 8 & $\mathrm{~N} 2$ & $4-15$ & 9.43 & $5.49 *$ & 24.85 & 20.32 & 66.85 & 3.23 & 34.22 \\
\hline 9 & N3 & $5-16$ & 9.38 & $4.31 *$ & 22.13 & 16.82 & 57.77 & 2.47 & 26.34 \\
\hline 10 & M1 & $4-13$ & $8.42 *$ & $3.99 *$ & 23.72 & 17.50 & 54.39 & 2.24 & 26.58 \\
\hline 11 & M2 & $3-11$ & $7.40 *$ & $4.15^{*}$ & 27.53 & 20.63 & 56.14 & 2.36 & 31.84 \\
\hline 12 & M3 & $3-16$ & $7.87 *$ & $5.35 *$ & 29.39 & 23.87 & 65.98 & 3.14 & 39.95 \\
\hline 13 & GE2 & $4-13$ & 9.00 & $6.81 *$ & 29.00 & 24.82 & 73.27 & 3.94 & 43.77 \\
\hline 14 & GN2 & $4-15$ & 9.05 & $7.05 *$ & 29.34 & 25.27 & 74.18 & 4.06 & 44.84 \\
\hline 15 & GM2 & $3-13$ & 8.65 & $6.53 *$ & 29.54 & 25.09 & 72.13 & 3.80 & 43.90 \\
\hline \multirow[t]{2}{*}{16} & Control & $7-13$ & 9.52 & 1.82 & & & & & \\
\hline & $\mathrm{CD}(5 \%)$ & - & 1.06 & 1.91 & & & & & \\
\hline \multicolumn{10}{|c|}{ OBGG-52 } \\
\hline 1 & G1 & $5-14$ & 8.48 & $5.38 *$ & 27.35 & 22.41 & 67.10 & 3.21 & 37.81 \\
\hline 2 & $\mathrm{G} 2$ & $5-15$ & 8.25 & $5.34 *$ & 28.01 & 22.90 & 66.85 & 3.18 & 38.58 \\
\hline 3 & G3 & $5-15$ & 7.87 & $5.28 *$ & 29.20 & 23.81 & 66.48 & 3.15 & 39.98 \\
\hline 4 & E1 & $5-15$ & 8.17 & $6.21 *$ & 30.50 & 25.79 & 71.50 & 3.67 & 44.92 \\
\hline 5 & E2 & $5-14$ & 8.17 & $6.21 *$ & 30.50 & 25.79 & 71.50 & 3.67 & 44.92 \\
\hline 6 & E3 & $4-14$ & $7.05 *$ & $5.30 *$ & 32.65 & 26.65 & 66.60 & 3.16 & 44.80 \\
\hline 7 & N1 & $5-14$ & 8.35 & $5.68 *$ & 28.54 & 23.68 & 68.84 & 3.38 & 40.47 \\
\hline 8 & $\mathrm{~N} 2$ & $4-15$ & 8.18 & $5.57 *$ & 28.85 & 23.83 & 68.22 & 3.32 & 40.55 \\
\hline 9 & N3 & $4-13$ & 7.53 & $5.21 *$ & 30.31 & 24.63 & 66.03 & 3.10 & 41.23 \\
\hline 10 & M1 & $4-14$ & $7.32 *$ & 3.83 & 26.74 & 19.61 & 53.79 & 2.17 & 29.62 \\
\hline 11 & M2 & $4-12$ & $6.75^{*}$ & 2.91 & 25.27 & 15.82 & 39.18 & 1.38 & 20.39 \\
\hline 12 & M3 & $4-09$ & $6.45^{*}$ & 1.94 & 21.59 & 6.39 & 8.76 & 0.25 & 3.90 \\
\hline 13 & GE2 & $5-13$ & 7.63 & $4.61 *$ & 28.14 & 22.09 & 61.61 & 2.72 & 35.71 \\
\hline 14 & GN2 & $4-13$ & 7.50 & 4.25 & 27.49 & 21.00 & 58.35 & 2.48 & 33.04 \\
\hline 15 & GM2 & $4-14$ & $7.17 *$ & 4.15 & 28.41 & 21.52 & 57.35 & 2.41 & 33.57 \\
\hline \multirow[t]{2}{*}{16} & Control & $6-11$ & 8.55 & 1.77 & & & & & \\
\hline & $\mathrm{CD}(5 \%)$ & - & 1.12 & 2.60 & & & & & \\
\hline
\end{tabular}

* Significant decrease (in mean) or increase (in variance) over control at $5 \%$ level.

Table.3 Genetic parameters for seeds per pods in different mutagenic treatment in $\mathrm{M}_{2}$ population 
of greengram

\begin{tabular}{|c|c|c|c|c|c|c|c|c|c|}
\hline $\begin{array}{l}\text { Tr. } \\
\text { No. }\end{array}$ & $\begin{array}{c}\text { Tr. } \\
\text { Code }\end{array}$ & Range & Mean & Variance & $\begin{array}{l}\text { PCV } \\
(\%)\end{array}$ & $\begin{array}{c}\text { GCV } \\
(\%)\end{array}$ & $\begin{array}{c}h^{2} \\
(\%)\end{array}$ & $\begin{array}{c}\text { GA } \\
(5 \%)\end{array}$ & $\begin{array}{c}\text { GA (\% of } \\
\text { Mean) }\end{array}$ \\
\hline \multicolumn{10}{|c|}{ Sujata } \\
\hline 1 & G1 & $6.3-9.9$ & 8.16 & 1.16 & 13.20 & 7.75 & 34.48 & 0.77 & 9.38 \\
\hline 2 & $\mathrm{G} 2$ & $5.3-9.9$ & $7.90 *$ & $1.72 *$ & 16.60 & 12.40 & 55.81 & 1.51 & 19.09 \\
\hline 3 & G3 & $5.4-10.0$ & 8.13 & $1.79 *$ & 16.46 & 12.48 & 57.54 & 1.59 & 19.51 \\
\hline 4 & E1 & $6.0-10.4$ & 8.27 & $1.67 *$ & 15.63 & 11.53 & 54.49 & 1.45 & 17.54 \\
\hline 5 & E2 & $5.8-9.8$ & 7.92 & $1.70 *$ & 16.46 & 12.24 & 55.29 & 1.49 & 18.75 \\
\hline 6 & E3 & $5.5-9.8$ & 7.96 & $1.68 *$ & 16.28 & 12.05 & 54.76 & 1.46 & 18.37 \\
\hline 7 & N1 & $6.0-10.5$ & 8.15 & $1.70 *$ & 16.00 & 11.90 & 55.29 & 1.49 & 18.22 \\
\hline 8 & N2 & $5.2-10.2$ & $7.60 *$ & $1.70 *$ & 17.16 & 12.76 & 55.29 & 1.49 & 19.54 \\
\hline 9 & N3 & $5.4-9.8$ & $7.61 *$ & $1.72 *$ & 17.23 & 12.88 & 55.81 & 1.51 & 19.81 \\
\hline 10 & M1 & $3.5-9.9$ & $7.73 *$ & $1.73 *$ & 17.02 & 12.74 & 56.07 & 1.52 & 19.65 \\
\hline 11 & M2 & $5.0-9.9$ & $7.46^{*}$ & $1.79 *$ & 17.93 & 13.60 & 57.54 & 1.59 & 21.26 \\
\hline 12 & M3 & $5.0-10.0$ & $7.44 *$ & $2.22 *$ & 20.03 & 16.24 & 65.77 & 2.02 & 27.13 \\
\hline 13 & GE2 & $5.3-9.8$ & $7.28 *$ & $1.77 *$ & 18.27 & 13.80 & 57.06 & 1.56 & 21.48 \\
\hline 14 & GN2 & $5.7-10.5$ & $7.85 *$ & 0.89 & 12.02 & 4.59 & 14.61 & 0.28 & 3.62 \\
\hline 15 & GM2 & $4.9-9.4$ & $7.01 *$ & $1.35^{*}$ & 16.57 & 10.96 & 43.70 & 1.05 & 14.92 \\
\hline \multirow[t]{2}{*}{16} & Control & $6.4-9.8$ & 8.55 & 0.76 & & & & & \\
\hline & $\mathrm{CD}(5 \%)$ & - & 0.64 & 0.44 & & & & & \\
\hline \multicolumn{10}{|c|}{ OBGG-52 } \\
\hline 1 & G1 & $6.4-11.6$ & 8.30 & 0.87 & 11.24 & 9.25 & 67.82 & 1.30 & 15.70 \\
\hline 2 & G2 & $6.5-11.2$ & 8.19 & $1.23^{*}$ & 13.54 & 11.90 & 77.24 & 1.76 & 21.55 \\
\hline 3 & G3 & $6.0-11.2$ & 8.15 & $1.53 *$ & 15.18 & 13.72 & 81.70 & 2.08 & 25.54 \\
\hline 4 & E1 & $5.5-11.4$ & 8.28 & $1.28 *$ & 13.66 & 12.08 & 78.13 & 1.82 & 21.99 \\
\hline 5 & $\mathrm{E} 2$ & $5.7-11.5$ & 8.31 & $1.34 *$ & 13.93 & 12.39 & 79.10 & 1.89 & 22.70 \\
\hline 6 & E3 & $6.5-10.7$ & 8.13 & $1.05^{*}$ & 12.6 & 10.79 & 73.33 & 1.55 & 19.04 \\
\hline 7 & N1 & $6.3-10.5$ & 8.29 & 0.72 & 10.24 & 8.00 & 61.11 & 1.07 & 12.89 \\
\hline 8 & $\mathrm{~N} 2$ & $6.4-10.7$ & 8.21 & 1.00 & 12.18 & 10.34 & 72.00 & 1.48 & 18.07 \\
\hline 9 & N3 & $4.7-11.3$ & 8.49 & $1.31 *$ & 13.48 & 11.95 & 78.63 & 1.85 & 21.84 \\
\hline 10 & M1 & $6.3-10.6$ & 8.24 & 0.54 & 8.92 & 6.19 & 48.15 & 0.73 & 8.85 \\
\hline 11 & M2 & $6.0-11.0$ & 7.88 & $1.39 *$ & 14.96 & 13.37 & 79.86 & 1.94 & 24.61 \\
\hline 12 & M3 & $5.4-09.8$ & $7.45 *$ & $1.21 *$ & 14.77 & 12.94 & 76.86 & 1.74 & 23.38 \\
\hline 13 & GE2 & $4.3-09.2$ & $6.90 *$ & 0.75 & 12.55 & 9.94 & 62.67 & 1.12 & 16.20 \\
\hline 14 & GN2 & $4.7-10.0$ & $6.91 *$ & $1.47 *$ & 17.55 & 15.79 & 80.95 & 2.02 & 29.26 \\
\hline 15 & GM2 & $5.5-09.2$ & $6.89 *$ & 0.61 & 11.34 & 8.34 & 54.10 & 0.87 & 12.63 \\
\hline \multirow[t]{2}{*}{16} & Control & $7.4-09.6$ & 8.51 & 0.28 & & & & & \\
\hline & $\mathrm{CD}(5 \%)$ & - & 0.86 & 0.67 & & & & & \\
\hline
\end{tabular}

* Significant decrease (in mean) or increase (in variance) over control at $5 \%$ level.

Table.4 Genetic parameters for 100-seed weight in different mutagenic treatment 
in $\mathrm{M}_{2}$ population of greengram

\begin{tabular}{|c|c|c|c|c|c|c|c|c|c|}
\hline $\begin{array}{l}\text { Tr. } \\
\text { No. }\end{array}$ & $\begin{array}{c}\text { Tr. } \\
\text { Code }\end{array}$ & Range & $\begin{array}{c}\text { Mean } \\
(\mathrm{gm})\end{array}$ & Variance & $\begin{array}{c}\text { PCV } \\
(\%)\end{array}$ & $\begin{array}{c}\text { GCV } \\
(\%)\end{array}$ & $\begin{array}{c}\mathbf{h}^{2} \\
(\%)\end{array}$ & $\begin{array}{c}\text { GA } \\
(5 \\
\%)\end{array}$ & $\begin{array}{c}\text { GA }(\% \\
\text { of } \\
\text { Mean })\end{array}$ \\
\hline \multicolumn{10}{|c|}{ Sujata } \\
\hline 1 & G1 & $2.26-3.45$ & 2.63 & $0.061 *$ & 9.39 & 6.48 & 47.54 & 0.24 & 9.20 \\
\hline 2 & $\mathrm{G} 2$ & $2.13-3.48$ & 2.59 & $0.081^{*}$ & 11.52 & 9.22 & 64.04 & 0.39 & 15.20 \\
\hline 3 & G3 & $2.10-3.16$ & 2.57 & $0.091^{*}$ & 11.72 & 9.41 & 64.44 & 0.40 & 15.56 \\
\hline 4 & E1 & $2.20-3.54$ & 2.63 & $0.066^{*}$ & 8.65 & 5.28 & 37.25 & 0.17 & 6.64 \\
\hline 5 & E2 & $2.01-3.41$ & 2.53 & $0.081^{*}$ & 10.72 & 8.04 & 56.16 & 0.31 & 12.40 \\
\hline 6 & E3 & $2.03-3.27$ & 2.52 & $0.085^{*}$ & 10.62 & 7.87 & 54.93 & 0.30 & 12.01 \\
\hline 7 & N1 & $2.06-3.18$ & 2.56 & $0.083^{*}$ & 10.67 & 8.04 & 56.76 & 0.32 & 12.47 \\
\hline 8 & $\mathrm{~N} 2$ & $2.04-3.14$ & 2.52 & $0.087^{*}$ & 10.73 & 8.00 & 55.56 & 0.31 & 12.28 \\
\hline 9 & N3 & $2.14-3.51$ & 2.52 & $0.100 *$ & 10.86 & 8.10 & 55.56 & 0.31 & 12.43 \\
\hline 10 & M1 & $2.02-3.10$ & 2.60 & $0.070 *$ & 10.18 & 7.50 & 54.29 & 0.30 & 11.38 \\
\hline 11 & M2 & $1.65-2.89$ & $2.27^{*}$ & $0.067^{*}$ & 11.32 & 8.12 & 51.52 & 0.27 & 12.01 \\
\hline 12 & M3 & $1.52-2.74$ & $1.97 *$ & $0.067 *$ & 11.41 & 6.72 & 34.69 & 0.16 & 8.15 \\
\hline 13 & GE2 & $1.79-2.87$ & $2.18^{*}$ & 0.053 & 9.67 & 5.05 & 27.27 & 0.12 & 5.43 \\
\hline 14 & GN2 & $2.15-2.98$ & 2.59 & $0.064^{*}$ & 9.77 & 6.91 & 50.00 & 0.26 & 10.06 \\
\hline 15 & GM2 & $2.15-3.21$ & 2.54 & $0.070 *$ & 9.88 & 6.93 & 49.21 & 0.25 & 10.02 \\
\hline \multirow[t]{2}{*}{16} & Control & $2.28-2.96$ & 2.67 & 0.030 & & & & & \\
\hline & $\mathrm{CD}(5 \%)$ & - & 0.15 & 0.031 & & & & & \\
\hline \multicolumn{10}{|c|}{ OBGG-52 } \\
\hline 1 & G1 & $2.36-3.59$ & 3.09 & 0.072 & 8.68 & 4.80 & 30.56 & 0.17 & 5.47 \\
\hline 2 & $\mathrm{G} 2$ & $2.54-3.69$ & $3.03 *$ & $0.085^{*}$ & 9.62 & 6.17 & 41.18 & 0.25 & 8.16 \\
\hline 3 & G3 & $2.58-3.78$ & $3.03 *$ & $0.097 *$ & 10.28 & 7.15 & 48.45 & 0.31 & 10.26 \\
\hline 4 & E1 & $2.42-3.73$ & $3.03 *$ & $0.086^{*}$ & 9.68 & 6.26 & 41.86 & 0.25 & 8.35 \\
\hline 5 & E2 & $2.19-3.60$ & $3.02 *$ & $0.107^{*}$ & 10.83 & 7.91 & 53.27 & 0.36 & 11.89 \\
\hline 6 & E3 & $2.11-3.53$ & $2.73 *$ & $0.086^{*}$ & 10.74 & 6.95 & 41.86 & 0.25 & 9.26 \\
\hline 7 & N1 & $2.46-3.48$ & $2.91 *$ & $0.087 *$ & 10.14 & 6.61 & 42.53 & 0.26 & 8.88 \\
\hline 8 & $\mathrm{~N} 2$ & $2.30-3.73$ & $2.84 *$ & $0.087 *$ & 10.39 & 6.77 & 42.53 & 0.26 & 9.10 \\
\hline 9 & N3 & $2.34-3.53$ & $2.86^{*}$ & $0.086^{*}$ & 10.25 & 6.63 & 41.86 & 0.25 & 8.84 \\
\hline 10 & M1 & $2.33-3.76$ & $2.89 *$ & $0.121^{*}$ & 12.04 & 9.22 & 58.68 & 0.42 & 14.55 \\
\hline 11 & M2 & $2.28-3.61$ & $2.88 *$ & $0.113^{*}$ & 11.67 & 8.72 & 55.75 & 0.39 & 13.41 \\
\hline 12 & M3 & $2.23-3.55$ & $2.78 *$ & $0.119 *$ & 12.41 & 9.45 & 57.98 & 0.41 & 14.82 \\
\hline 13 & GE2 & $2.18-3.76$ & $2.80 *$ & $0.120 *$ & 12.37 & 9.45 & 58.33 & 0.42 & 14.87 \\
\hline 14 & GN2 & $2.26-3.68$ & $2.85 *$ & $0.103^{*}$ & 11.26 & 8.08 & 51.46 & 0.34 & 11.94 \\
\hline 15 & GM2 & $2.10-3.80$ & $2.75^{*}$ & 0.076 & 10.02 & 5.86 & 34.21 & 0.19 & 7.06 \\
\hline 16 & Control & $2.85-3.57$ & 3.22 & 0.050 & & & & & \\
\hline & $\mathrm{CD}(5 \%)$ & - & 0.14 & 0.035 & & & & & \\
\hline
\end{tabular}

* Significant decrease (in mean) or increase (in variance) over control at $5 \%$ level.

Table.5 Genetic parameters for yield per plant in different mutagenic treatment 
in $\mathrm{M}_{2}$ population of greengram

\begin{tabular}{|c|c|c|c|c|c|c|c|c|c|}
\hline $\begin{array}{l}\text { Tr. } \\
\text { No. }\end{array}$ & $\begin{array}{c}\text { Tr. } \\
\text { Code }\end{array}$ & Range & $\begin{array}{c}\text { Mean } \\
\text { (gm) }\end{array}$ & Variance & $\begin{array}{c}\text { PCV } \\
(\%)\end{array}$ & $\begin{array}{c}\text { GCV } \\
(\%)\end{array}$ & $\begin{array}{c}h^{2} \\
(\%)\end{array}$ & $\begin{array}{c}\text { GA } \\
(5 \%)\end{array}$ & $\begin{array}{c}\text { GA (\% } \\
\text { of Mean) }\end{array}$ \\
\hline \multicolumn{10}{|c|}{ Sujata } \\
\hline 1 & G1 & $0.95-3.91$ & 2.05 & $0.51 *$ & 34.84 & 28.44 & 66.67 & 0.98 & 47.84 \\
\hline 2 & $\mathrm{G} 2$ & $0.71-3.64$ & 1.93 & $0.51 *$ & 38.52 & 32.27 & 70.18 & 1.09 & 55.68 \\
\hline 3 & G3 & $0.77-3.92$ & $1.85^{*}$ & $0.55^{*}$ & 39.72 & 32.88 & 68.52 & 1.04 & 56.07 \\
\hline 4 & E1 & $0.85-3.66$ & $1.89 *$ & $0.35^{*}$ & 29.13 & 19.18 & 43.33 & 0.49 & 26.01 \\
\hline 5 & $\mathrm{E} 2$ & $0.56-3.63$ & $1.73 *$ & $0.41 *$ & 38.34 & 30.04 & 61.36 & 0.84 & 48.47 \\
\hline 6 & E3 & $0.79-3.53$ & $1.63 *$ & $0.40 *$ & 37.04 & 26.91 & 52.78 & 0.65 & 40.27 \\
\hline 7 & N1 & $0.87-3.63$ & 1.96 & 0.23 & 23.50 & 10.26 & 19.05 & 0.18 & 9.22 \\
\hline 8 & $\mathrm{~N} 2$ & $0.56-3.12$ & $1.77 *$ & 0.21 & 27.99 & 15.12 & 29.17 & 0.29 & 16.82 \\
\hline 9 & N3 & $0.87-3.78$ & $1.83^{*}$ & $0.34 *$ & 34.50 & 25.91 & 56.41 & 0.73 & 40.09 \\
\hline 10 & M1 & $0.65-3.64$ & $1.70 *$ & $0.33^{*}$ & 32.05 & 20.62 & 41.38 & 0.46 & 27.32 \\
\hline 11 & M2 & $0.30-2.20$ & $1.26 *$ & 0.20 & 35.49 & 13.75 & 15.00 & 0.14 & 10.97 \\
\hline 12 & M3 & $0.38-2.78$ & $1.16^{*}$ & 0.22 & 37.90 & 12.30 & 10.53 & 0.09 & 8.22 \\
\hline 13 & GE2 & $0.48-2.92$ & $1.45^{*}$ & $0.33^{*}$ & 41.38 & 30.06 & 52.78 & 0.65 & 44.99 \\
\hline 14 & GN2 & $0.89-3.60$ & $1.84 *$ & $0.38 *$ & 35.86 & 28.09 & 61.36 & 0.84 & 45.32 \\
\hline 15 & GM2 & $0.52-3.04$ & $1.52 *$ & 0.25 & 31.76 & 16.22 & 26.09 & 0.26 & 17.07 \\
\hline \multirow[t]{2}{*}{16} & Control & $1.42-2.74$ & 2.16 & 0.12 & & & & & \\
\hline & $\mathrm{CD}(5 \%)$ & - & 0.26 & 0.14 & & & & & \\
\hline \multicolumn{10}{|c|}{ OBGG-52 } \\
\hline 1 & G1 & $1.07-3.94$ & 2.16 & $0.36^{*}$ & 27.78 & 22.20 & 63.89 & 0.79 & 36.56 \\
\hline 2 & G2 & $1.22-3.82$ & 2.03 & $0.37 *$ & 29.96 & 24.13 & 64.86 & 0.81 & 40.04 \\
\hline 3 & G3 & $0.95-3.78$ & $1.93 *$ & $0.40^{*}$ & 32.77 & 26.92 & 67.50 & 0.88 & 45.57 \\
\hline 4 & E1 & $0.87-3.96$ & 2.02 & $0.35^{*}$ & 29.29 & 23.22 & 62.86 & 0.77 & 37.92 \\
\hline 5 & $\mathrm{E} 2$ & $0.84-3.61$ & 2.03 & $0.39 *$ & 30.76 & 25.12 & 66.67 & 0.86 & 42.25 \\
\hline 6 & E3 & $0.73-3.47$ & $1.58 *$ & $0.39 *$ & 39.53 & 32.27 & 66.67 & 0.86 & 54.28 \\
\hline 7 & N1 & $0.89-3.42$ & 2.01 & $0.37 *$ & 30.26 & 24.37 & 64.86 & 0.81 & 40.44 \\
\hline 8 & $\mathrm{~N} 2$ & $0.87-3.76$ & $1.93 *$ & $0.51 *$ & 37.00 & 31.94 & 74.51 & 1.10 & 56.79 \\
\hline 9 & N3 & $0.79-4.09$ & $1.86^{*}$ & $0.56^{*}$ & 40.23 & 35.26 & 76.79 & 1.18 & 63.64 \\
\hline 10 & M1 & $1.02-4.16$ & $1.74 *$ & $0.35^{*}$ & 34.00 & 26.96 & 62.86 & 0.77 & 44.03 \\
\hline 11 & M2 & $0.62-3.16$ & $1.53 *$ & 0.23 & 31.35 & 20.67 & 43.48 & 0.43 & 28.07 \\
\hline 12 & M3 & $0.50-2.58$ & $1.35^{*}$ & 0.20 & 33.13 & 19.60 & 35.00 & 0.32 & 23.88 \\
\hline 13 & GE2 & $0.84-2.74$ & $1.46^{*}$ & 0.17 & 28.24 & 13.70 & 23.53 & 0.20 & 13.69 \\
\hline 14 & GN2 & $0.59-3.76$ & $1.48 *$ & 0.29 & 36.39 & 27.03 & 55.17 & 0.61 & 41.35 \\
\hline 15 & GM2 & $0.57-3.64$ & $1.37 *$ & 0.23 & 35.01 & 23.08 & 43.48 & 0.43 & 31.35 \\
\hline \multirow[t]{2}{*}{16} & Control & $1.55-2.85$ & 2.33 & 0.13 & & & & & \\
\hline & $\mathrm{CD}(5 \%)$ & - & 0.33 & 0.17 & & & & & \\
\hline
\end{tabular}

* Significant decrease (in mean) or increase (in variance) over control at $5 \%$ level.

Yield per plant (Table 5) 
Mean yield per plant in treated populations of Sujata varied from $1.16 \mathrm{gm}$ (M3) to $2.05 \mathrm{gm}$ (G1) whereas $2.16 \mathrm{gm}$ in control and in the case of OBGG-52, it ranged from $1.35 \mathrm{gm}$ (M3) to $2.16 \mathrm{gm}$ (G1) whereas $2.33 \mathrm{gm}$ in control. All mutagenic treatments showed lower seed yield than respective controls. Mutagenic treatments except for G1, G2 and $\mathrm{N} 1$ in Sujata and G1, G2, E1, E2 and N1 in OBGG-52 showed a significant negative shift of mean as compared to respective controls. Though there was no definite trend, the reduction was generally higher in higher doses (except N3 of Sujata) and in combined treatments. Yield per plant was with a variation of 1.42 to $2.74 \mathrm{gm}$ and 1.55 to $2.85 \mathrm{gm}$ in control population of Sujata and OBGG-52, respectively. But the variation in yield/plant in the mutagenic treated populations was much wider with a range of 0.30 to $3.92 \mathrm{gm}$ in Sujata and 0.50 to $4.16 \mathrm{gm}$ in OBGG-52. This trait for different treated populations of Sujata and OBGG-52 exhibited a variance range of $0.20(\mathrm{M} 2)$ to $0.55(\mathrm{G} 3)$ and 0.17 (GE2) to $0.56(\mathrm{~N} 3)$, respectively, while in the respective controls the variances were 0.12 and 0.13 . All mutagenic treatments of both the varieties exhibited higher population variance than respective controls. All the mutagenic treatments, except N1, N2, M2, M3, GM2 in Sujata and M2, M3 GE2, GN2, GM2 in OBGG-52, showed a significant increase in population variance as compared to their parent respective controls.

The PCV estimates for this trait in different treated populations of Sujata and OBGG-52 ranged from $23.50(\mathrm{~N} 1)$ to 41.38 (GE2) and 27.78 (G1) to $40.23(\mathrm{~N} 3)$, respectively. The GCV estimates for yield in the treatments ranged from $10.26 \%(\mathrm{~N} 1)$ to $32.88 \%(\mathrm{G} 3)$ in Sujata and $13.70 \%$ (GE2) to $35.26 \%(\mathrm{~N} 3)$ in OBGG-52 and it was relatively higher $(\geq$ 25\%) in G1, G2, G3, E2, E3 N3, GE2and GN2 treatments of Sujata and G3, E2, E3, N2, N3, M1and GN2 treatments of OBGG-52.
Heritability estimates for yield in treated populations varied from $10.53 \%$ (M3) to $70.18 \%(\mathrm{G} 2)$ in Sujata and $23.53 \%$ (GE2) to $76.79 \%(\mathrm{~N} 3)$ in OBGG-52. Heritability estimates were higher in G1, G2, G3, E2, E3, N3, GE2 and GN2 treatment poulations of Sujata and G1, G2, G3, E1, E2, E3. N1, N2, N3, M1 and GN2 of OBGG-52. Genetic advance in the treatments varied from 0.09 (M3) to 1.09 (G2) in Sujata and 0.20 (GE2) to 1.18 (N3) in OBGG-52. GA as a percentage of mean in treatment population varied from $8.22 \%(\mathrm{M} 3)$ to $56.07 \%(\mathrm{G} 3)$ and $13.69 \%$ (GE2) to $63.64 \%(\mathrm{~N} 3)$ in the case of Sujata and OBGG-52, respectively and it was generally moderate to high in both varieties.

Induction of mutation is an important tool for the creation of genetic variability for specific characters in a crop when the variability of the crop is completely exhausted and can lead to no further possibility for genetic improvement through the conventional approach. In mutation breeding, the possibility exists to change a single gene or only a few genes without altering the total genetic makeup of a specific/outstanding genotype (Broertjes and Van Harten, 1978).

In this study, there was a negative shift in mean for all these quantitative characters in mutagen treated $\mathrm{M}_{2}$ populations in both the varieties and the shift was significant in most of the cases. A large portion of this induced variability in different treatments might have been due to the induction of more micromutations with a negative effect on the trait. Thus mean seed yield in the treatments was lower than the control. A wide range of variation and lower population mean of the treatments showed that induction of change was more in a negative direction and less in a positive direction. A similar differential negative shift of mean in different $\mathrm{M}_{2}$ populations of greengram was presented by Das and Misra (2005), Das et al. (2006), Khan et al. (2006), and Kozgar et al. (2011). 
In most of the above reports, the shift of mean varied with mutagens and their doses. In this study, there was a greater reduction in higher doses for most traits. A decrease in the mean value of various quantitative traits at higher concentrations may be due to mutagenic treatment means was shifted to a direction opposite to selection (Bhatia and Swaminathan, 1962). A comparative study of the effect of mutagens indicated that the negative shift of mean was more pronounced in $\mathrm{MH}$ and its combined treatments in both the varieties. This might be due to chromosomal abnormalities like stickiness, fragments, laggards, bridges, etc (Ghose and Chatterjee, 1989). The comparatively lower magnitude of a negative shift in gamma rays treatments might be due to less drastic effect and either induction of less number of micro mutants or induction of micro mutations in either direction (Das and Misra, 2005). The negative shift could be attributed to either physiological damage caused chiefly by chemical mutagens or chromosomal aberrations caused mainly by irradiations (Swaminathan et al., 1968; Brock, 1971). Induction of more chromosomal aberrations was also reported by Virk et al., (1978) in NG than EMS treatments.

Most of the mutagen treated $\mathrm{M}_{2}$ populations showed a significant increase in population variance than the control population for all the characters studied in both of the varieties. Among different treatments each mutagen group sufficient genetic variability observed in the induced population of G3, E3, N2, M3 and GN2 for pods/plant; G3, E2, N3, M3 and GE2 for seeds/pod; G3, E3, N3, M1 and GM2 for 100- seed weight and G3, E2, N3, M1 and GN2 for yield/plant in Sujata whereas, G1, E1, E2, N1 and GE2 for pods/plant; G3, E2, N3, M2 and GN2 for seeds/pod; G3, E2, N2, M1 and GE2 for 100-seed weight and G3, E3, $\mathrm{N} 3$ and M1 for yield/plant in OBGG-52. The increase in the variance of the $\mathrm{M}_{2}$ population in a trait is a general indicator of the induction of micro mutation with a negative and/or positive effect on the trait. However, the magnitude of an increase in population variance varied with the mutagen and their concentration, parental genotype and the character concerned. A similar increase in variability in $\mathbf{M}_{2}$ population and differential increase in treatments with different mutagens, their combinations and doses in various growth and yield attributing traits in greengram had earlier been reported by Das and Misra (2005), Das et al. (2006), Khan et al. (2006), Wani and Khan (2006) and Kozgar et al. (2011). The study showed that though the dose-variance relationship was not completely linear, in most of the cases higher doses of mutagens induced greater variance. These results are in agreement with earlier reports of Singh et al. (2001) and Sharma et al. (2008).

In General, most mutagens treated $\mathrm{M}_{2}$ populations showed an increase in phenotypic variability which can be attributed partly to genetic effects and partly to environmental effects. Depending upon the magnitude of induced genetic variability in different treatment populations, different genetic parameters like the phenotypic coefficient of variability (PCV), genetic coefficient of variability $(\mathrm{GCV})$, heritability and genetic advance under selection (GA) would vary with different mutagens and its doses. These parameters represent an indication of the effectiveness of mutagenic treatments for the induction of micro-mutations and the scope of improvement for the trait through selection in $\mathrm{M}_{2}$ populations.

The GCV estimates varied with mutagenic treatments and were high (> 20\%) for pods/plant and yield per plant while moderate (10-20\%) for seeds/pod and low $(<10 \%)$ for 100 -seed weight in both varieties. Similar results for different traits in greengram were 
also reported earlier by Das et al. (2006), Singh et al. (2001), Sharma et al. (2008) and Kozgar et al. (2010, 2011). The GCV value alone is not enough to determine the amount of heritable variability thus estimation of heritability and genetic advance along with the GCV are required to assess the heritable portion of the total variation. Heritability along with high GA has indicated the existence of additive and epistatic gene effects, which are fixable and can provide the desirable genetic gain. Thus, it is necessary to consider all these three genetic parameters (GCV, heritability and genetic advance) for an effective selection of a particular trait.

The heritability estimates for different traits in $\mathrm{M}_{2}$ populations varied with mutagens and their dose and in many cases it was relatively high in both varieties for all traits, indicating the greater scope of improvement through selection in those treated population. Genetic advance as a percentage of mean also increased in treatments and comparatively higher for pods/plant and yield per plant of both the varieties. Similar results for different traits in mutagenic populations of greengram were also reported by Das and Misra (2005), Mishra et al. (2008) and Sharma et al. (2008).

In conclusion, the present investigation most of the mutagenic treatments induced a wide range of variation in populations than their parental population in both varieties. The range indicated that almost all mutagenic treatments induced a wider range of variation in $\mathrm{M}_{2}$ populations in both directions for all the traits. Most treatment populations exhibited a reduction in population mean and increased in population variance for all the four traits studied and the magnitude of such changes varied with mutagens, their dose and the variety indicating that these treatments appeared to be effective for induction of micro mutation in yield component traits however higher dose of gamma-rays and NG, moderate to a higher dose of EMS, the low dose of $\mathrm{MH}$ and moderate dose combination of gamma rays and $\mathrm{NG}$ found as the most useful dose for creation of sufficient induced genetic variability for yield as well as the yield attributing characters. The selection of these doses of different mutagens would be effective in the creation of sufficient genetic variability and the development of desirable high yielding genotypes. The higher value of GCV, heritability and genetic advance for yield and yield attributing traits i.e. pods per plant, seeds per pod and 100 seed weight indicated that selection in $\mathrm{M}_{2}$ populations would be effective for yield improvement.

\section{References}

Anonymous. 2018. Directorate of Economics and Statistics (DES), 2018.// eands.dacnet.nic.in

Bhatia, C. R. and Swaminathan, M.S. (1962). Induced polygenic variability in bread wheat and it's bearing on selection procedure. Z. pflanzenauecht., 48: 317326.

Brock, R.D.1971. The role of induced mutation in plant improvement. Radiat. Bot., 11:181-196.

Broertjes, C. and Van Harten, A.M. 1978. Application of Mutation Breeding Methods in the Improvement of Vegetatively Propagated Crops. Eisevier Sei. Pub. Co., Amsterdam.

Das T. R. and Baisakh B. (2019) Selection indices and discriminant function analysis for grain yield in greengram [Vigna radiata (L.) Wilczek]. e-planet 17(1):13-21.

Das, T. R. and Misra, R. C. 2005. Genetic analysis of mutagen induced variability in yield traits in greengram (Vigna radiata). Environment and Ecology, 23(2): 381-384.

Das, T. R., Misra, R. C. and Sahu, P. K. 2006. 
Efficiency of mutagenic treatments in expression of macro and micro mutations in $\mathbf{M}_{2}$ generation in greengram and its early predictability on basis of $\mathrm{M}_{1}$ parameters. Environment and Ecology, 24(2): 283-288.

Ghose, S. K. and Chatterjee, P. K. 1989. EMS, Hydrazine hydrate, Dimethyl sulfoxide induced mutations in jute. Proc. 76th Indian Sci. Cong. III (X):7pp.

Khan, S., Wani, M. R. and Praveen, K. 2006. Quantitative variability in mungbean induced by chemical mutagens. Legume Res., 29(2): 143-145.

Kozgar, M. I., Goyal, S. and Khan, S. 2011. EMS induced mutational variability in Vigna radiata and $V$. mungo. Res. J. Botany, 6: 31-37.

Mishra, L. D., Baisakh, B. and Nayak, P. K. 2008. Genetic variability among advanced mutant lines of mungbean. Journal of Food Legumes, 21(4): 274275.

Sharma, V., Kumar, G. and Kuamr, R. 2008. EMS induced polygenic variations in mungbean. Journal of Food Legumes, 21(1): 61-62.

Singh, G., Sareen, P. K., Saharan, R. P. and Singh, A. 2001. Induced variability in mungbean ( $V$. radiata (L.) Wilczek). Indian J. Genet., 61: 281-282.

Swaminathan, M. S., Siddiq, E. A. S., Savin, V. N. and Varughese, G. 1968. Studies on enhancement of mutation frequency and identification of mutations in plantbreeding and phylogenetic significance of some cereals. In: Mutations in Plant Breeding II, IAEA, Vienna, and 233$248 \mathrm{pp}$.

Virk, D. S., Saini, S. S. and Gupta, V. P. 1978. Gamma radiation induced polygenic variation in pure breeding and segregating genotypes of wheat and rice. Env. Expt. Bot, 18: 185-191.

Wani, M. R. and Khan, S. 2006. Estimates of genetic variability in mutated populations and the scope of selection for yield attributes in Vigna radiata (L.) Wilczek. Egyptian Journal of Biology, 8: 1-6.

\section{How to cite this article:}

Das, T. R., Baisakh B. and Prusti, A.M. 2020. Induced Genetic Variability and the Scope of Selection for Yield Attributes in Greengram (Vigna radiata L. Wilczek). Int.J.Curr.Microbiol.App.Sci. 9(04): 2988-3000. doi: https://doi.org/10.20546/ijcmas.2020.904.350 